\title{
Capnocytophaga sputigena
}

National Cancer Institute

\section{Source}

National Cancer Institute. Capnocytophaga sputigena. NCI Thesaurus. Code C86242.

A species of facultatively anaerobic, Gram-neg ative, fusiform, filamentous bacilli assigned to the phylum Bacteroidetes. This species is motile, non-spore forming, catalase, oxidase, urease and indole negative, reduces nitrate, hydrolyzes gelatin and does not produce hydrogen sulfide. C. sputigena is a member of the normal gingival flora but can become pathogenic causing sepsis especially in immunocompromised individuals. 